\title{
Formation of potential titanium antigens based on protein binding to titanium dioxide nanoparticles
}

\author{
Carmen Irina Vamanu' \\ Paul Johan $\mathrm{Høl}{ }^{2}$ \\ Zouhir Ekeland Allouni \\ Said Elsayed ${ }^{3}$ \\ Nils Roar Gjerdet' \\ 'Departments of Oral Sciences - \\ Dental Biomaterials, Faculty of \\ Dentistry, University of Bergen, \\ Bergen, Norway; ${ }^{2}$ Orthopedic \\ Biomaterials, Helse Bergen Haukeland \\ University Hospital, Bergen, Norway; \\ ${ }^{3}$ Department of Medicine, University \\ of Bergen, Bergen, Norway
}

\begin{abstract}
Degradation products of titanium implants include free ions, organo-metallic complexes, and particles, ranging from nano to macro sizes. The biological effects, especially of nanoparticles, is yet unknown. The main objective of this study was to develop Ti-protein antigens in physiological solutions that can be used in testing of cellular responses. For this purpose, $0.1 \% \mathrm{TiO}_{2}$ nanoparticles less than $100 \mathrm{~nm}$ were mixed with human serum albumin (HSA), $0.1 \%$ and $1 \%$, in cell culture medium (DMEM, pH 7.2). The Ti concentrations in the resulting solutions were analyzed by inductively coupled plasma mass spectrometry. The stability of the nanoparticles in suspension was analyzed by UV-vis spectrophotometer and Dynamic Light Scattering. The concentration of Ti in suspension was dependent on the presence and concentration of HSA. Albumin prevented high aggregation rate of $\mathrm{TiO}_{2}$ nanoparticles in cell culture medium. It is shown that nano $\mathrm{TiO}_{2}$-protein stable aggregates can be produced under physiological conditions at high concentrations, and are candidates for use in cellular tests.

Keywords: titanium, nanoparticle, hypersensitivity to $\mathrm{Ti}$
\end{abstract}

\section{Introduction}

Titanium (Ti) is widely used in medicine and dentistry, either in its pure form or in alloys, due to the combination of physical, chemical and biological properties (Scharnweber et al 2002). Titanium is employed in eg, joint prostheses, fracture fixation devices, endovascular stents, pacemaker enclosures, and dental materials (Peters et al 1984; Yamauchi et al 2000; Stoeckel et al 2004; Tschernitschek et al 2005).

The high corrosion resistance of $\mathrm{Ti}$ is due to a strongly passivating oxide layer (Hallab et al 2004). Nevertheless, Ti degrades under mechanical stress, abrasion, low $\mathrm{pH}$ and low oxygen level (Tschernitschek et al 2005). It is established that Ti-based implants release particles and ions to the surrounding tissue and fluids, as has been demonstrated with hip implants (Mckellop et al 1990; Brien et al 1992), as well as with miniplates used for jaw fractures (Schliephake et al 1993; Torgersen et al 1995), and even unloaded dental implants (Arys et al 1998; Flatebø et al 2006). However, the biological consequences of the degradation products, ions, nano- or micrometer sized particles, remain unclarified.

Based mostly on case reports, it has been suggested that hypersensitivity to $\mathrm{Ti}$ is associated with implant loosening (Wooley et al 1996; Matthew and Frame 1998), or skin reactions (Peters et al 1984; Yamauchi et al 2000; Tamai et al 2001; High et al 2006; Thomas et al 2006; Watanabe et al 2006). Diagnosis of contact allergy to metals usually relies on combination of anamnestic information and in vivo tests such as a epicutaneous patch test (Hallab et al 2001; Granchi et al 2006), and - in some instances - ex vivo tests such as a Lymphocyte Stimulation Test (LST) on cells from blood samples (Hallab et al 2001). Hypersensitivity tests have not been established for Ti (Lalor et al 1991; Hallab et al 2001; Klein et al 2004) and Ti is 
not included in standard commercial routine patch test series. Various titanium compounds, such as Ti-salts (Lalor et al 1991) and $\mathrm{TiO}_{2}$ micrometer sized particles (Thomas et al 2006) have been tried in hypersensitivity testing, but optimal Ti antigens are not established yet (Bircher and Stern 2001).

The ex vivo test, LST, requires cell culture techniques and bioavailable $\mathrm{Ti}$ at physiological culture conditions (Hallab et al 2001). The difficulty with $\mathrm{Ti}$ is that this metal is not readily soluble at physiological $\mathrm{pH}$, as nickel salts are (Lalor et al 1991; Hallab et al 2000; Hallab et al 2004). Had relevant and stable Ti-substances been available, the applicability of patch testing (Granchi et al 2006) and LST (Hallab et al 2001) could be improved for Ti.

Nanoparticles, with dimensions less than $100 \mathrm{~nm}$, interact differently with various proteins and subsequently with cells when compared with the conventional size particles (Liu and Webster 2006). Surface functionalization of gold nanoparticles has been used to develop diagnostic and treatment tools (Shenoy et al 2006). Surface modification of nanoparticles can be achieved by protein binding.

Albumin has been shown to be dominant in binding $\mathrm{Ti}$ in vivo (Hallab et al 2000). It is the most prevalent protein in plasma (approximately $42 \mathrm{~g} / 1$ for adults) with important functions such as metal binding, transport, and in providing colloidal osmotic pressure (Vander et al 1998).

By simulating the model of metal-protein binding in vivo, the aim of the present study was to develop Ti-protein antigens based on titanium dioxide nanoparticles that can be useful for testing cellular responses in diagnosis of human Ti hypersensitivity.

\section{Materials and methods Materials}

Commercially pure titanium dioxide $\left(\mathrm{TiO}_{2}\right)$ nanoparticles, $99.9 \%$, consisting of the crystallographic forms anatase and rutile (MW05084, Sigma-Aldrich, St. Louis, USA) were used. The powder particles have an average diameter $<100 \mathrm{~nm}$. The specific surface area of the $\mathrm{TiO}_{2}$ nanopowder, determined by the Brunauer-Emmet-Teller (BET) method according to the nitrogen adsorption (ASAP 2020 Volumetric Adsorption, Micromeritics), was $19.6 \mathrm{~m}^{2} / \mathrm{g}$. The cell culture medium used was Dulbecco's Modified Eagle's Medium (DMEM, Sigma-Aldrich, D6046, UK). The medium is a high ionic strength solution and contains low amounts of glucose and amino acids. Commercially available human serum albumin (HSA) (96\%-99\%, Sigma-Aldrich, SL04521, St. Louis, USA) was used.

\section{Methods}

\section{Preparation of potential antigens and determination} of Ti-concentrations

Several suspensions were prepared by mixing the reagents in DMEM. A suspension was prepared by mixing $50 \mathrm{mg} \mathrm{TiO}_{2}$ in $50 \mathrm{ml}$ DMEM for a concentration of $0.1 \% \mathrm{TiO}_{2}$. Different quantities of HSA - $50 \mathrm{mg}(0.1 \%)$ and $500 \mathrm{mg}$ (1\%) - were added. Control suspensions of $0.1 \% \mathrm{TiO}_{2}$ nanoparticles in DMEM ( $0 \%$ HSA) were prepared. The suspensions were placed in a shaking machine in an incubator at $37^{\circ} \mathrm{C}$ for 8 days in order to assess the effect of exposure time between nano- $\mathrm{TiO}_{2}$ and $\mathrm{HSA}$. Aliquots of $2 \mathrm{ml}$ were taken from each solution, after $1 \mathrm{~h}, 24 \mathrm{~h}$, and 8 days. The samples were centrifuged at $3000 \mathrm{rpm}$ (15 min), and sterile filtered (Millex HV filters, Millipore, Carrigtwohill, Co. Cork, Ireland, pore size $0.45 \mu \mathrm{m})$. The assays ran 6 times.

The total concentration of titanium in the filtered suspensions was determined Inductively Coupled Plasma - Mass Spectrometry (ICP-MS). The instrument (Element 2, Thermo Finnigan, Bremen, Germany) used a high resolution, magnetic sector field. Colloidal suspensions of nanoparticles with average sizes of $400 \mathrm{~nm}$ have been shown to be totally ionized in the argon plasma, which holds a temperature of nearly $8000 \mathrm{~K}$ (Degueldre and Favarger 2003). The instrument was operated at medium resolution $(\mathrm{R}=4000)$ for the isotopes Ti47 and Ti49, and the resulting signal count at the mass detector was calibrated to represent the total Ti-concentration in the sample. The sample aliquots $(0.5 \mathrm{ml})$ were diluted by a factor of 100-200 with ultra-clean 1\% nitric acid before measurements. An internal standard of indium $(1 \mu \mathrm{g} / \mathrm{l})$ was added to all the samples to monitor and to correct for any instrumental fluctuations. Calibration was performed by standard addition using calibrating solutions made from a $1000 \mathrm{mg} / \mathrm{l}$ stock standard solution (Merck, Darmstadt, Germany). The method detection limit for the Ti analysis was $0.56 \mu \mathrm{g} / \mathrm{l}$. The accuracy of the analytical method was monitored by a reference material (Seronorm Trace Elements Serum level 1, lot MI0181, Sero AS, Billingstad, Norway) with a recommended Ti-value of $2.6 \mu \mathrm{g} / \mathrm{l}$. We found a median value of $2.55(\mathrm{n}=10)$.

\section{Transmission electron microscopy (TEM)}

Suspensions with $0.1 \% \mathrm{TiO}_{2}$ nanoparticles in DMEM only and in DMEM + $0.1 \%$ HSA were prepared at the same conditions (shaking machine; incubator $37^{\circ} \mathrm{C}$ ) as previously mentioned. The suspensions were shaken for 4 days. The samples prepared for TEM images were not centrifuged and sterile filtered in order to visualize the $\mathrm{TiO}_{2}$ nanoparticles and aggregate formation. One drop $(10 \mu \mathrm{l})$ from each suspension, 
diluted 10 times, was placed on a $\mathrm{Cu}$ mesh grid. The excess liquid was removed immediately by filter paper. The samples were lyophilized overnight at room temperature, and analyzed using a transmission electron microscope (JEM-1230) at electron emission of $60 \mathrm{kV}$.

\section{Physicochemical characterization: Stability of $\mathrm{TiO}_{2}$ nanoparticles in suspension}

Dynamic light scattering (DLS) was used to measure the hydrodynamic diameter of aggregates in suspension. The particles in suspension are subject to the thermal Brownian motion and the rate of particles movement depends upon their size (Tkachenko et al 2006). Suspensions of $0.1 \% \mathrm{TiO}_{2}$ in DMEM and $\mathrm{TiO}_{2}$ with $0.1 \%$ and $1 \%$ of HSA in DMEM were prepared, shaken for 2 hours, ultrasonicated for $30 \mathrm{sec}$, and analyzed. The measurements were conducted on the Zetasizer NS (Malvern Instruments Ltd, UK).

The $\mathrm{TiO}_{2}$ nanoparticles sedimentation rate was determined by monitoring the absorbance at $337 \mathrm{~nm}$ (the wavelength of maximum absorbance of the solid) as a function of time by UV-vis spectrophotometer (Perkin-Elmer, Model 554 , Germany). The $\mathrm{TiO}_{2}$ suspensions were prepared in the same manner as described for DLS study and analyzed before and after sterile filtration (pore size $0.45 \mu \mathrm{m}$ ). All measurements were made at $25^{\circ} \mathrm{C}$. Square cuvettes with $1 \mathrm{~cm}$ light path were used. The center of the light beam passed through the cuvette $1.5 \mathrm{~cm}$ above its bottom.

\section{Statistical analysis}

Statistical analysis was performed using SPSS version 13.0. P-values less than or equal to 0.05 were considered significant. Friedman test was used for time-line differences and Mann-Whitney test for the two levels of albumin concentrations.

\section{Results}

The preparation of antigens in the first part of our study was proceeded for a period of 8 days, in orders to asses the effect of the exposure time between nano- $\mathrm{TiO}_{2}$ and albumin on $\mathrm{Ti}$ concentration. Based on obtained results, the study continued with TEM imaging and the determination of stability of nano- $\mathrm{TiO}_{2}$ antigens in suspension.

The filtered suspensions, with no HSA, gave a low median Ti concentration (by ICP-MS), below $1 \mu \mathrm{g} / \mathrm{l}$, and unaffected by exposure time. The presence of HSA increased the concentration of Ti by 2-3 orders of magnitude when compared to controls (suspensions without HSA). Longer exposure time raised also the Ti concentrations in suspensions with 0.1 and

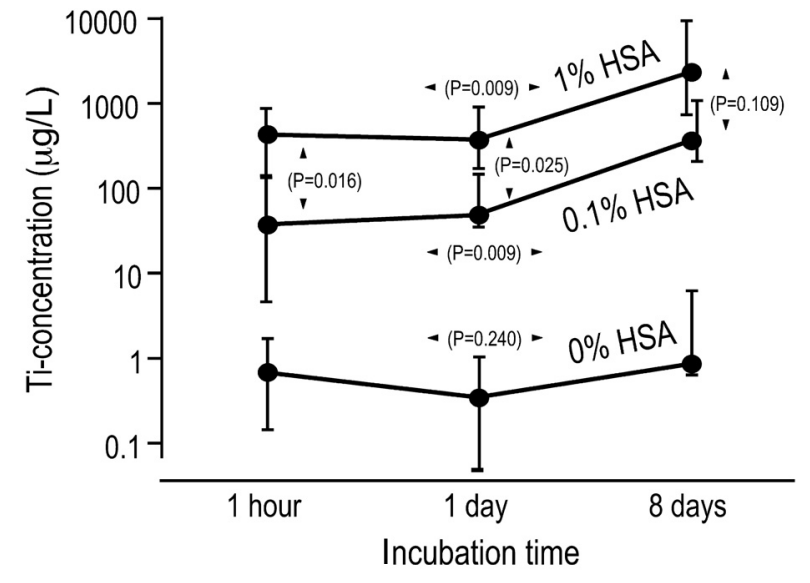

Figure I Ti concentration as a function of incubation time (median, whiskers represent quartiles) in DMEM after mixing $\mathrm{TiO}_{2}$ nanoparticles with different concentrations of human serum albumin (HSA). $\mathrm{P}$-values with vertical arrows: comparisons between the results with 0.1 and I\% HSA; p-values with horizontal arrows: Friedman's test across the time points.

1\% HSA (Figure 1). Differences between Ti concentrations with $0.1 \% \mathrm{HSA}$ and $1 \% \mathrm{HSA}$ were statistically different for all time points, except at day 8 .

The TEM images of the unfiltered suspensions revealed that the individual $\mathrm{TiO}_{2}$ nanoparticles were spherical, with diameters ranging from 20 to more than $100 \mathrm{~nm}$. The $\mathrm{TiO}_{2}$ nanoparticles formed typical aggregates in both suspensions, with and without albumin (Figures $2 \mathrm{a}$ and $2 \mathrm{~b}$ ).

The DLS measurements results showed that $\mathrm{TiO}_{2}$ nanoparticles formed large aggregates in DMEM without HSA, but the mean aggregate size was about $1 / 5$ in presence of HSA. After filtration, the mean aggregate size was about half of the unfiltered suspensions (Table 1). The evolution of $\mathrm{TiO}_{2}$ aggregates size in DMEM with $1 \% \mathrm{HSA}$ revealed a slow growth over $20 \mathrm{~h}$ period (regression model: mean size $(\mathrm{nm})=1.46$ time $\left.(\mathrm{h})+210 ; \mathrm{R}^{2}=0.83\right)$.

The sedimentation rate results revealed that the $\mathrm{TiO}_{2}$ aggregates sedimented rapidly ( $5 \mathrm{~h}$ ) in absence of HSA, whereas in presence of HSA, the sedimentation rate was close to zero (Figure 3). After filtration, the $\mathrm{TiO}_{2}$ aggregates were stable in suspension all along a $20 \mathrm{~h}$ period (Figure 3 ). In DMEM without HSA, after filtration, the $\mathrm{TiO}_{2}$ nanoparticles were not detectable by either Zetasizer or the UV-vis spectrophotometer.

\section{Discussion}

The results presented in this study show that the combination of human serum albumin and Ti in the form of nanoparticles raises the bioavailability of $\mathrm{Ti}$ in suspension, most likely through a metal-protein association. 

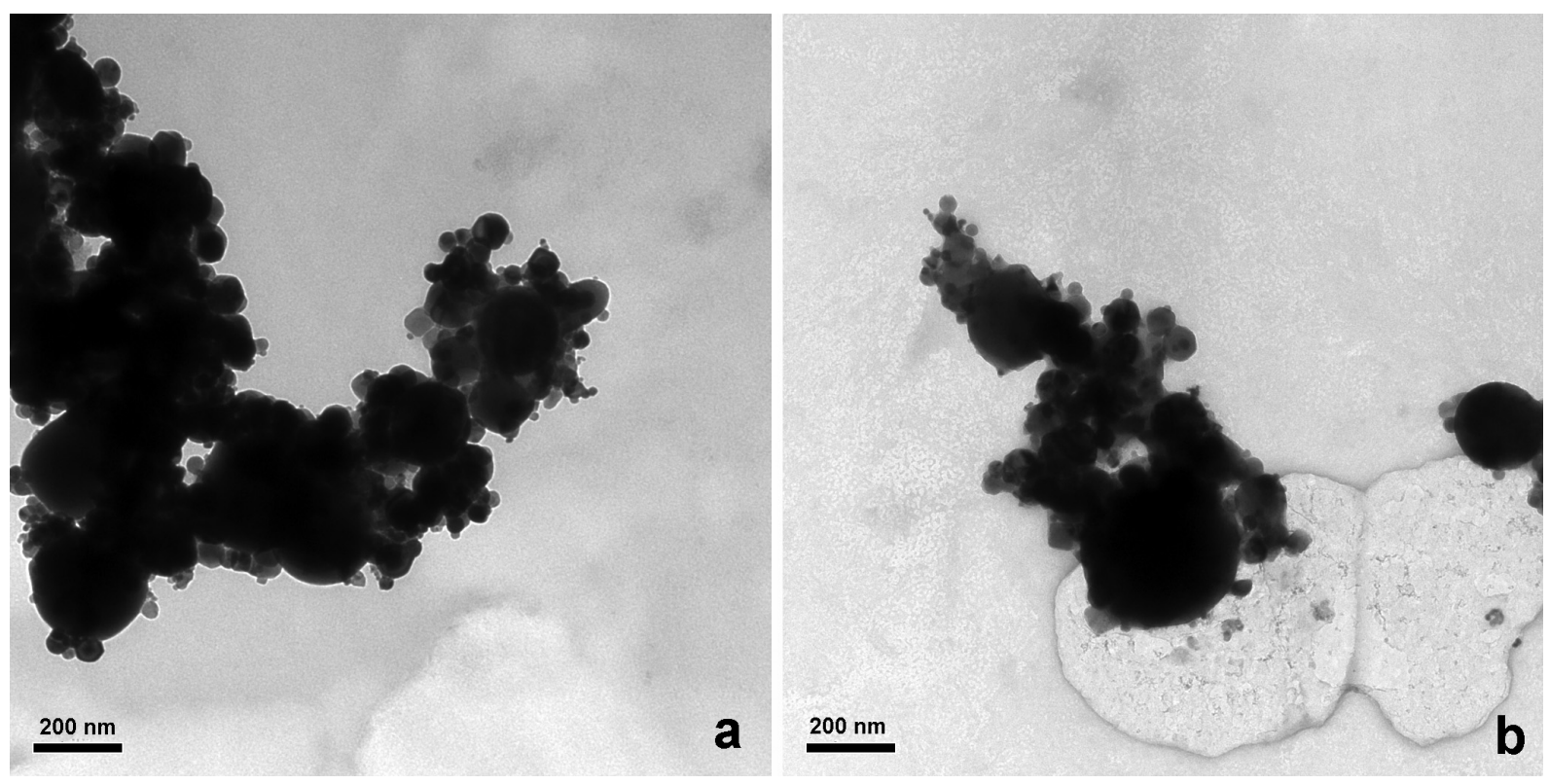

Figure 2 Transmission electron microscopy (TEM) images of typical $\mathrm{TiO}_{2}$ nanoparticle aggregates in DMEM. TiO ${ }_{2}$ nanoparticles aggregate in both suspensions without (a) and with $0.1 \%$ human serum albumin (HSA) (b). Pictures are taken from unfiltered suspensions, due to the difficulty of finding the characteristic aggregates in filtered suspensions.

Using nanoparticles to develop a potential diagnostic reagent for hypersensitivity testing is new and clinically appropriate because nanometer sized wear products have been found in tissues surrounding implants (Buscher et al 2005). Titanium dioxide nanoparticles have greater potential toxic effect on cells than the conventional size particles (Chen et al 2006; Long et al 2006).

When compared with the micrometer-sized and easily detectable particles, the nanoparticles have larger specific surfaces and different physiochemical properties, which mean higher surface energy (Long et al 2006). Consequently, their interaction with cell membrane, cell structure, or cell activity may differ from the larger-size particulate matter (Long et al 2006).

Our results suggest that $\mathrm{TiO}_{2}$ nanoparticles, which aggregate and sediment rapidly in cell culture medium, are more stable in suspensions when they are in presence of HSA. This increased stability could be due to protein adsorption onto the surface of $\mathrm{TiO}_{2}$ nanoparticles or aggregates (Klinger et al 1997; Oliva et al 2003). Moreover, steric repulsive forces caused by the protein chain reduce the net attractive interactions between particles, and thus alter the aggregation rate (Tirado-Miranda et al 2003; Limbach et al 2005). The presence of albumin slowed down the aggregate settling as shown by the sedimentation rate test, and raised the Ti concentration orders of magnitude even after filtration.
In presence of HSA, the mean aggregate size, as revealed by DLS, was slightly smaller than the pore size of $450 \mathrm{~nm}$ and thus most of the aggregates passed through the filter membrane (Table 1). These results are in concordance with results from the quantitative chemical analyses (ICP-MS) showing high final Ti concentration in the samples (Figure 1). Furthermore, the effect of HSA on the reducing the aggregate size of $\mathrm{TiO}_{2}$ nanoparticles in suspensions is supported by our sedimentation study (Figure 3). Ultrasonication used for $30 \mathrm{sec}$ of $\mathrm{TiO}_{2}$-albumin suspensions showed also an effect on clustering size, by breaking down the big aggregates.

Few studies have been done on the colloidal stability of nanoparticles in cell culture media. Our results are in accordance with Long et al (2006) who revealed that DMEM affects the rate of aggregation and consequently the size of clusters. In solution with high ionic strength without proteins, such as DMEM medium, the rate of aggregation is high, leading to

Table I Mean hydrodynamic diameter for $\mathrm{TiO}_{2}$ after $2 \mathrm{~h}$ of shaking $(n=3)$

\begin{tabular}{lll}
\hline Sample & \multicolumn{2}{l}{ Mean hydrodynamic diameter $(\mathbf{n m}) \pm$ SD } \\
\cline { 2 - 3 } & Before filtration & After filtration \\
\hline $\mathrm{TiO}_{2} / \mathrm{DMEM}$ & $2765 \pm 124$ & Not detected \\
$\mathrm{TiO}_{2}+0.1 \%$ & $446 \pm 26$ & $215 \pm 1.7$ \\
$\mathrm{HSA}^{\prime} \mathrm{DMEM}$ & & \\
$\mathrm{TiO}_{2}+1 \%$ & $430 \pm 23$ & $216 \pm \mathrm{I}$ \\
$\mathrm{HSA} / \mathrm{DMEM}$ & & \\
\hline
\end{tabular}




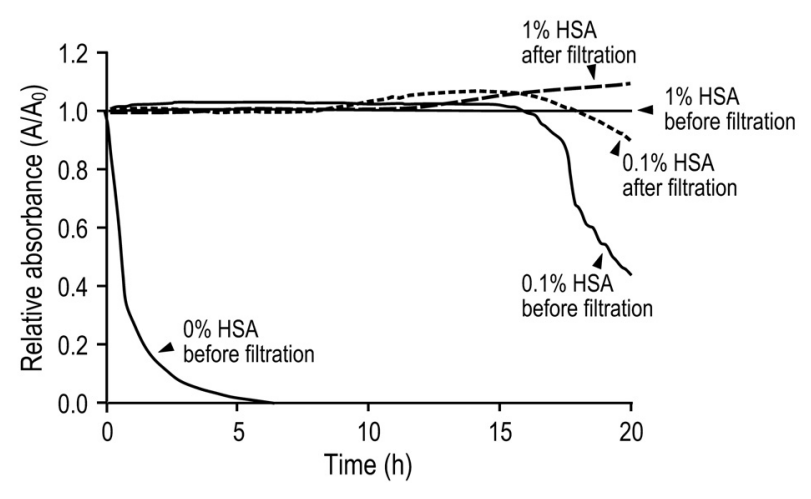

Figure 3 Sedimentation of $\mathrm{TiO}_{2}$ nanoparticles in DMEM with different concentrations of human serum albumin (HSA), before and after filtration $\left(A_{0}\right.$ represents absorption at $\mathrm{t}=0$ ).

fast sedimentation (Gomez-Lopera et al 2006; Phenrat et al 2007). However, if HSA is present, the adsorption of the protein onto $\mathrm{TiO}_{2}$ surfaces is almost independent of the ionic strength (Oliva et al 2003). The binding mechanisms of the $\mathrm{TiO}_{2}$-albumin interaction need more research.

It can be assumed that the $\mathrm{TiO}_{2}$ nanoparticles behave in a similar manner in vivo, making aggregates and binding to proteins. This supports the idea that the metal released by implant degradation does not exist as unbound ionic or colloidal forms in serum but bound to serum proteins (Hallab et al 2000).

Since our potential antigens could be prepared already after $2 \mathrm{~h}$ of shaking, we recommend using these fresh suspensions, in hypersensitivity testing, instead of those mixed for 8 days.

The biological reactivity of the new possible antigens is now being pursued in Lymphocyte Stimulation Test (LST). More tests needs to be done in order to standardize the method.

\section{Conclusions}

The $\mathrm{TiO}_{2}$ nanoparticle-albumin antigen could be clinically relevant as it mimics degradation products of Ti-based implants. Albumin has an important role in the colloidal stability of $\mathrm{TiO}_{2}$ nanoparticles in suspension, and thus, on the bioavailability of Ti for cellular tests. The applicability of the substances in clinical testing of hypersensitivity has to be elucidated.

\section{Acknowledgments}

Chief engineer Egil Erichsen (Electron Microscopy Laboratory, University of Bergen), and senior engineer Trygve Knag and research engineer Anne Nyhaug (MIC center, The Department of Biomedicine, Haukeland University Hospital, Bergen) have contributed with valuable technical assistance concerning the scanning and transmission electron microscopy.

\section{Conflicts of interest}

None.

\section{References}

Arys A, Philippart C, Dourov N, et al. 1998. Analysis of titanium dental implants after failure of osseointegration: combined histological, electron microscopy, and X-ray photoelectron spectroscopy approach. J Biomed Mater Res, 43:300-12.

Bircher AJ, Stern WB. 2001. Allergic contact dermatitis from "titanium" spectacle frames. Contact Dermatitis, 45:244-5.

Brien WW, Salvati EA, Betts F, et al. 1992. Metal levels in cemented total hip arthroplasty. A comparison of well-fixed and loose implants. Clin Orthop Relat Res, 66-74.

Buscher R, Tager G, Dudzinski W, et al. 2005. Subsurface microstructure of metal-on-metal hip joints and its relationship to wear particle generation. J Biomed Mater Res B Appl Biomater, 72:206-14.

Chen HW, Su SF, Chien CT, et al. 2006. Titanium dioxide nanoparticles induce emphysema-like lung injury in mice. Faseb $J$.

Degueldre C, Favarger PY. 2003. Colloid analysis by single particle inductively coupled plasma-mass spectroscopy: a feasibility study. Colloids Surf A Physicochem Eng Aspects, 217:137-42.

Flatebø RS, Johannessen AC, Grønningsæter AG, et al. 2006. Host response to titanium dental implant placement evaluated in a human oral model. J Periodontol, 77:1201-10.

Gomez-Lopera SA, Arias JL, Gallardo V, et al. 2006. Colloidal stability of magnetite/poly(lactic acid) core/shell nanoparticles. Langmuir, 22:816-21.

Granchi D, Cenni E, Trisolino G, et al. 2006. Sensitivity to implant materials in patients undergoing total hip replacement. $J$ Biomed Mater Res $B$ Appl Biomater, 77:257-64.

Hallab N, Jacobs J, Katz J. 2004. Orthopedic applications. In: Ratner B, Hoffman A, Schoen F, et al eds. Biomaterial science. An introduction to materials in medicine. 2nd ed. Amsterdam: Elsevier Academic Press. p 537.

Hallab N, Merritt K, Jacobs JJ. 2001. Metal sensitivity in patients with orthopaedic implants. J Bone Joint Surg Am, 83-A:428-36.

Hallab NJ, Anderson S, Caicedo M, et al. 2004. Immune responses correlate with serum-metal in metal-on-metal hip arthroplasty. J Arthroplasty, 19:88-93.

Hallab NJ, Jacobs JJ, Skipor A, et al. 2000. Systemic metal-protein binding associated with total joint replacement arthroplasty. J Biomed Mater Res, 49:353-61.

Hallab NJ, Mikecz K, Jacobs JJ. 2000. A triple assay technique for the evaluation of metal-induced, delayed-type hypersensitivity responses in patients with or receiving total joint arthroplasty. J Biomed Mater Res, 53:480-9.

Hallab NJ, Mikecz K, Vermes C, et al. 2001. Differential lymphocyte reactivity to serum-derived metal-protein complexes produced from cobalt-based and titanium-based implant alloy degradation. $J$ Biomed Mater Res, 56:427-36.

High WA, Ayers RA, Adams JR, et al. 2006. Granulomatous reaction to titanium alloy: an unusual reaction to ear piercing. $J$ Am Acad Dermatol, 55:716-20.

Klein R, Schwenk M, Heinrich-Ramm R, et al. 2004. Diagnostic relevance of the lymphocyte transformation test for sensitization to beryllium and other metals - (IUPAC Technical Report. Pure Appl Chem, 76:1269-81.

Klinger A, Steinberg D, Kohavi D, et al. 1997. Mechanism of adsorption of human albumin to titanium in vitro. $J$ Biomed Mater Res, 36:387-92.

Lalor PA, Revell PA, Gray AB, et al. 1991. Sensitivity to titanium. A cause of implant failure?. J Bone Joint Surg Br, 73:25-8. 
Limbach LK, Li YC, Grass RN, et al. 2005. Oxide nanoparticle uptake in human lung fibroblasts: Effects of particle size, agglomeration, and diffusion at low concentrations. Environ Sci Technol, 39:9370-6.

Liu H, Webster TJ. 2006. Nanomedicine for implants: a review of studies and necessary experimental tools. Biomaterials, 28:354-69.

Long TC, Saleh N, Tilton RD, et al. 2006. Titanium dioxide (P25) produces reactive oxygen species in immortalized brain microglia (BV2): implications for nanoparticle neurotoxicity. Environ Sci Technol, 40:4346-52.

Matthew I, Frame JW. 1998. Allergic responses to titanium. J Oral Maxillofac Surg, 56:1466-7.

Mckellop HA, Sarmiento A, Schwinn CP, et al. 1990. In vivo wear of titanium-alloy hip prostheses. J Bone Joint Surg Am, 72A:512-17.

Oliva FY, Avalle LB, Camara OR, et al. 2003. Adsorption of human serum albumin (HSA) onto colloidal $\mathrm{TiO}_{2}$ particles, Part I. J Colloid Interf Sci, 261:299-311.

Peters MS, Schroeter AL, van Hale HM, et al. 1984. Pacemaker contact sensitivity. Contact Dermatitis, 11:214-18.

Phenrat T, Saleh N, Sirk K, et al. 2007. Aggregation and sedimentation of aqueous nanoscale zerovalent iron dispersions. Environ Sci Technol, 41:284-90.

Scharnweber D, Beutner R, Rossler S, et al. 2002. Electrochemical behavior of titanium-based materials - are there relations to biocompatibility? J Mater Sci Mater Med, 13:1215-20.

Schliephake H, Lehmann H, Kunz U, et al. 1993. Ultrastructural findings in soft-tissues adjacent to titanium plates used in jaw fracture treatment. Int J Oral Maxillofac Surg, 22:20-5.

Shenoy D, Fu W, Li J, et al. 2006. Surface functionalization of gold nanoparticles using hetero-bifunctional poly(ethylene glycol) spacer for intracellular tracking and delivery. Int J Nanomedicine, 1:51-8.
Stoeckel D, Pelton A, Duerig T. 2004. Self-expanding nitinol stents: material and design considerations. Eur Radiol, 14:292-301.

Tamai K, Mitsumori M, Fujishiro S, et al. 2001. A case of allergic reaction to surgical metal clips inserted for postoperative boost irradiation in a patient undergoing breast-conserving therapy. Breast Cancer, 8:90-2 .

Thomas P, Bandl WD, Maier S, et al. 2006. Hypersensitivity to titanium osteosynthesis with impaired fracture healing, eczema, and T-cell hyperresponsiveness in vitro: case report and review of the literature. Contact Dermatitis, 55:199-202.

Tirado-Miranda M, Schmitt A, Callejas-Fernandez J, et al. 2003. The aggregation behaviour of protein-coated particles: a light scattering study. Eur Biophys J, 32:128-36.

Tkachenko NH, Yaremko ZM, Bellmann C. 2006. Effect of 1-1-charged ions on aggregative stability and electrical surface properties of aqueous suspensions of titanium dioxide. Colloids Surf A Physicochem Eng Aspects, 279:10-19.

Torgersen S, Gjerdet NR, Erichsen ES, et al. 1995. Metal particles and tissue changes adjacent to miniplates. A retrieval study. Acta Odontol Scand, 53:65-71.

Tschernitschek H, Borchers L, Geurtsen W. 2005. Nonalloyed titanium as a bioinert metal-a review. Quintessence Int, 36:523-30.

Vander A, Sherman J, Luciano D. 1998. Human physiology. The mechanisms of body function. In: ed. 7th Edition. Boston: WCB McGrawHill. p. 374-5.

Watanabe R, Nanko H, Fukuda S. 2006. Lymphocytoma cutis due to pierced earrings. J Cutan Pathol, 33:16-19.

Wooley PH, Nasser S, Fitzgerald RH Jr. 1996. The immune response to implant materials in humans. Clin Orthop Relat Res, 63-70.

Yamauchi R, Morita A, Tsuji T. 2000. Pacemaker dermatitis from titanium. Contact Dermatitis, 42:52-3. 